\title{
Modulation of the Methionine Action on Serum Cholesterol and Lipoprotein Profiles by Dietary Carbohydrates in Normal, Hepatoma-bearing and Cholesterol-loaded Donryu Rats
}

\author{
Kazumi Yagasaki, Yutaka SeKIBA, Shoko InagaKi \\ and Ryuhei FunABIKI \\ Department of Agricultural Chemistry, Faculty of Agriculture, \\ Tokyo Noko University, Fuchu, Tokyo I83, Japan
}

Received March 10, 1989

\begin{abstract}
The methionine (Met) action on serum cholesterol (Ch) and lipoprotein profiles was studied by varying the dietary carbohydrate sources in normal, hepatoma (AH109A)-bearing and Ch-loaded Donryu rats fed on $20 \%$ casein diets for 2 weeks. In normal rats, Met $(1.8 \%)$ addition to a $20 \%$ casein diet containing a sucrose-starch $(1: 3)$ mixture increased HDL-Ch without affecting (VLDL + LDL)-Ch, and resulted in a significant reduction of the (VLDL + LDL)-Ch/HDL-Ch ratio (atherogenic index, $\mathrm{AI}$ ), while Met showed no significant influence on them under dietary condition of sucrose alone or starch alone. In hepatoma-bearing rats, Met (1.2\%) also had a significant influence only when the sucrose-starch mixture was used as the dietary carbohydrate source, the amino acid reducing $\mathrm{AI}$ by both suppressing the hepatoma-induced elevation in (VLDL + LDL)-Ch and increasing HDL-Ch. In Ch-loaded rats, however, Met $(0.75 \%)$ exerted no significant influence under the dietary carbohydrate source of the sucrose-starch mixture, but this amino acid significantly decreased (VLDL + LDL)-Ch without affecting HDL-Ch, and hence reduced AI under the dietary condition of sucrose alone or starch alone. These results suggest that dietary carbohydrates modulate the Met action on cholesterolemia depending on the absence or presence of dietary $\mathrm{Ch}$, and that Met is essentially antiatherogenic from the aspect of lipoprotein profiles when the amino acid expresses significant influence on cholesterolemia in Donryu rats.
\end{abstract}

There have been a number of studies concerning the action of dietary supplemented methionine (Met) on the serum cholesterol (Ch) concentration at standard casein levels $(20 \sim 25 \%)$. In animals fed on Ch-added diets, the effect of Met has been reported to be hypocholesterolemic in chicks ${ }^{11}$ and rats, ${ }^{2)}$ or hypercholesterolemic in rats. ${ }^{3)}$ Such fluctuating effects of Met have also been reported in animals fed on $\mathrm{Ch}$-free diets; either hypocholesterolemic in rats, ${ }^{4}$ hypercholesterolemic in rabbits ${ }^{57}$ and rats, ${ }^{6}$ or of no effect in rats. ${ }^{7,81}$ In endogenously hypercholesterolemic animals, Met has been reported to act as a hypocholesterolemic amino acid in hypothyroid rats ${ }^{9}$ and hepatoma-bearing rats. ${ }^{10}$ ) These discrepancies in the Met action on cholesterolemia are thought to be due to such facturs as differences in the level of supplemented Met, feeding period, quality and quantity of other dietary components than Met, animal species and strain, age, sex, hormonal and pathologic states, and the cause of hypercholesterolemia.

The purpose of the present study is to estimate whether or not dietary carbohydrate sources modulate the Met effect on serum $\mathrm{Ch}$ concentration and lipoprotein profiles, using rats in three states, i.e., normal (normocholesterolemic), hepatoma-bearing (endogenously hypercholesterolemic) and Ch-loaded (exogenously hypercholesterolemic). Starch, sucrose and a mixture of the two were selected as dietary carbohydrate sources, since they have been widely used in many studies.

\section{Materials and Methods}

Animals and diets. Male rats of the Donryu strain (Nippon Rats Co., Urawa, Saitama) were used throughout the experiments. The animals were initially fed on a stock 
Table I. Composition of the Basal Dieis (\%)

\begin{tabular}{lcc}
\hline Ingredient & $\begin{array}{c}\text { Cholesterol- } \\
\text { free diet }\end{array}$ & $\begin{array}{c}\text { Cholesterol- } \\
\text { enriched diet }\end{array}$ \\
\hline Carbohydrates & $67^{a}$ & $50.75^{b}$ \\
Casein $^{c}$ & 20 & 20 \\
Corn oil $^{d}$ & 5 & 5 \\
Mineral mixture $^{e}$ & 5 & 5 \\
Vitamin mixture $^{e}$ & 1 & 1 \\
Cellulose powder $^{c}$ & 2 & 2 \\
Lard $^{d}$ & - & 15 \\
Cholesterol $^{f}$ & - & 1 \\
Sodium cholate $^{f}$ & - & 0.25 \\
\hline
\end{tabular}

a Sucrose (Mitsui Sugar Co., Ltd., Tokyo), $\alpha$-corn starch ${ }^{c}$ or sucrose-starch (17:50) mixture.

$b$ Sucrose, $\alpha$-corn starch or sucrose-starch $(17: 33.75)$ mixture.

c Oriental Yeast Co., Ltd., Tokyo.

¿ Yuro Yakuhin Co., Ltd., Tokyo.

e Mineral mixture I and vitamin mixture 1 (Oriental arrangement).

$f$ Wako Pure Chemical Ind. Ltd., Osaka:

pellet (CE-2, CLEA Japan Inc., Tokyo) for 5 to 7 days in an air-conditioned room with an 8:00 a.m. to $8: 00$ p.m. light cycle. They were then divided into groups with equal body weights and fed on the experimental diets in individual cages with wire bottoms. The composition of the basal diets without $\mathrm{Ch}$ addition (experiments 1 and 2 ) and with $\mathrm{Ch}$ addition (experiment 3 ) expressed in percentage weight is shown in Table $I$. To these basal diets was added L-Met (Ajinomoto Co., Tokyo) at the expense of carbohydrates. In experiment 2 , the rats received a subcutaneous implantation of $5 \times 10^{5} \mathrm{AH} 109 \mathrm{~A}$ cells (provided by the Sasaki Institute, Tokyo) per rat to produce a solid tumor in the back as previously described. ${ }^{10.11)}$ The animals in all the experiments were fed on the experimental diets and water ad libitum for 14 days, and they were deprived of food at 9:00 a.m. (experiments 1 and 2) and 7:00 a.m. (experiment 3 ) on the 14 th day. The rats were allowed free access to water until sacrifice, which was conducted $4 \mathrm{hr}$ (experiments 1 and 2) and $8 \mathrm{hr}$ (experiment 3) later by decapitation. The blood was collected, left to clot and centrifuged to obtain serum. The liver or solid hepatoma was quickly removed, washed with cold $0.9 \% \mathrm{NaCl}$, blotted on filter paper and weighed.

Lipid analyses. Total lipids were extracted according to the procedure of Folch et al. ${ }^{12)}$ from the liver and solid hepatoma ( $c a .0 .5 \mathrm{~g}$ ). After aliquots of the chloroform phase had been dried, the total $\mathrm{Ch}(\mathrm{TCh})^{13)}$ and triglyceride $(\mathrm{TG})^{143}$ were determined as previously described ${ }^{15}$ The serum TG level was also determined. ${ }^{14}$

Lipoprotein separation and cholesterol determination. The serum lipoproteins were separated into high-density lipoprotein (HDL) and very-low-density lipoprotein + low-density lipoprotein (VLDL + LDL) fractions by the precipitation method. ${ }^{11)}$ The total contents of the unfractionated whole serum (TCh) and HDL were determined by an enzymatic method using a Cholesterol C-Test kit (Wako Pure Chemical Ind. Ltd., Osaka). The difference between $\mathrm{TCh}$ and $\mathrm{HDL}-\mathrm{Ch}$ is regarded as (VLDL+ LDL) $\mathrm{Ch}$.

Statistical method. A statistical analysis was carried out using Student's $t$ test between the control (without Met) and test (with Met) groups under each carbohydrate condition, and a $p$ value of $<0.05$ is considered significant.

\section{Results}

The effect of the Met supplement to Ch-free, $20 \%$ casein diets was first examined in normal (normocholesterolemic) rats by varying the dietary carbohydrate source (experiment 1 in Table II). The addition of $1.8 \%$ Met caused significant decreases in food intake and growth irrespective of the dietary carbohydrates, i.e., sucrose alone, starch alone and their mixture. No significant change, however, was observed in the relative liver weight between the control and Met-supplemented groups under any dietary carbohydrate condition. The serum lipid levels showed characteristic changes from Met addition, depending on the dietary carbohydrates: Met exerted no significant influence upon the serum TG, TCh, HDL $-\mathrm{Ch}$ and (VLDL + LDL)-Ch levels and hence atherogenic index $(\mathrm{AI})$ in the cases of sucrose alone and starch alone. However, with the sucrosestarch mixture, the amino acid significantly elevated the HDL-Ch level without affecting the (VLDL + LDL)-Ch level, and this led to a significant elevation in the $\mathrm{TCh}$ level with a significant reduction of AI. The serum TG level showed a decreasing tendency $(p<0.1)$ with Met addition in the case of the sucrosestarch mixture. The liver TCh and TG contents were unaffected by Met addition with all carbohydrate sources.

Hepatoma-bearing rats with abnormal lipoprotein profiles ${ }^{10,11)}$ were given $\mathrm{Ch}$-free diets, and the effect of Met $(1.2 \%)$ was examined in relation to the dietary carbohydrate source 
Table II. Effect of Methionine (1.8\%) Addition to the 20\% Casein Diets Containing Sucrose, Starch or a Sucrose-Starch Mixture on the Food Intake, Body Weight Gain, Liver Weight, and Serum and Liver Lipid Levels in Normal Donryu Rats (Experiment 1)

\begin{tabular}{|c|c|c|c|c|c|c|}
\hline \multirow{3}{*}{ Measurement } & \multicolumn{6}{|c|}{ Dietary carbohydrate source } \\
\hline & \multicolumn{2}{|c|}{ Sucrose } & \multicolumn{2}{|c|}{ Starch } & \multicolumn{2}{|c|}{ Sucrose-starch ${ }^{a}$} \\
\hline & Control & + Met & Control & + Met & Control & + Met \\
\hline No. of rats & 6 & 5 & 6 & 5 & 5 & 5 \\
\hline Initial body weight $(\mathrm{g})$ & $105.3 \pm 3.6$ & $107.4 \pm 4.1$ & $106.0 \pm 3.1$ & $107.5 \pm 2.6$ & $126.1 \pm 3.1$ & $126.8 \pm 4.0$ \\
\hline Food intake ( $\mathrm{g} / 2$ weeks) & $194.1 \pm 12.1$ & $138.6 \pm 5.1^{* *}$ & $212.3 \pm 9.4$ & $148.3 \pm 8.0^{* *}$ & $219.0 \pm 4.0$ & $174.3 \pm 3.3^{* *}$ \\
\hline Body weight gain (g/2 weeks) & $67.3 \pm 10.0$ & $30.9 \pm 3.7^{* *}$ & $65.3 \pm 7.0$ & $20.2 \pm 6.8^{* *}$ & $73.9 \pm 3.1$ & $44.9 \pm 3.1^{* *}$ \\
\hline $\begin{array}{l}\text { Liver weight } \\
(\mathrm{g} / 100 \mathrm{~g} \text { of body weight })\end{array}$ & $4.87 \pm 0.17$ & $4.51 \pm 0.22$ & $4.13 \pm 0.16$ & $4.05 \pm 0.14$ & $4.75 \pm 0.11$ & $4.91 \pm 0.20$ \\
\hline \multicolumn{7}{|c|}{ Serum lipid $(\mathrm{mg} / 100 \mathrm{ml}$ of serum) } \\
\hline Triglyceride & $82.5 \pm 8.1$ & $70.9 \pm 7.6$ & $65.4 \pm 5.3$ & $64.8 \pm 2.5$ & $68.5 \pm 8.7$ & $48.0 \pm 4.5$ \\
\hline Total cholesterol & $93.3 \pm 3.8$ & $95.7 \pm 8.6$ & $85.4 \pm 4.9$ & $98.9 \pm 5.2$ & $57.5 \pm 2.9$ & $88.0 \pm 3.2^{* *}$ \\
\hline $\mathrm{HDL}-\mathrm{Ch}(\mathrm{A})$ & $51.5 \pm 2.9$ & $46.9 \pm 4.8$ & $48.2 \pm 3.3$ & $54.7 \pm 3.1$ & $31.8 \pm 1.4$ & $61.7 \pm 5.4^{* *}$ \\
\hline (VLDL + LDL)-Ch (B) & $41.8 \pm 5.8$ & $48.8 \pm 4.7$ & $37.2 \pm 5.2$ & $44.7 \pm 4.8$ & $25.7 \pm 2.0$ & $26.4 \pm 4.6$ \\
\hline Altherogenic index $(\mathrm{B} / \mathrm{A})$ & $0.85 \pm 0.15$ & $1.06 \pm 0.09$ & $0.80 \pm 0.14$ & $0.84 \pm 0.11$ & $0.81 \pm 0.09$ & $0.47 \pm 0.12 *$ \\
\hline \multicolumn{7}{|l|}{ Liver lipid ( $\mathrm{mg} / \mathrm{g}$ of liver) } \\
\hline Total cholesterol & $2.49 \pm 0.05$ & $2.43 \pm 0.05$ & $2.54 \pm 0.14$ & $2.43 \pm 0.09$ & $2.43 \pm 0.05$ & $2.36 \pm 0.07$ \\
\hline Triglyceride & $12.5 \pm 2.3$ & $17.1 \pm 4.2$ & $11.6 \pm 2.2$ & $10.7 \pm 2.3$ & $13.6 \pm 2.2$ & $14.1 \pm 3.0$ \\
\hline
\end{tabular}

Each value represents the mean $\pm S E M$.

"An experiment on these two groups was conducted separately from that on the other four groups.

$* p<0.05$ and ${ }^{* *} p<0.005$ to the corresponding control groups.

(experiment 2 in Table III). In the case of hepatoma-bearing rats, unlike the normal ones, no significant suppression was observed in food intake and growth by Met addition with any carbohydrate source. The relative liver and hepatoma weights were also unaffected by Met addition, irrespective of the carbohydrate source. No significant effect was caused on serum lipid levels and AI by Met addition when the dietary carbohydrate sources were sucrose alone and starch alone. In the case of the sucrose-starch mixture, Met significantly lowered the (VLDL + LDL)-Ch level and elevated the $\mathrm{HDL}-\mathrm{Ch}$ level, thus resulting in an unchanged $\mathrm{TCh}$ level but with a significant reduction of $\mathrm{AI}$. The serum TG level was reduced by Met addition with this carbohydrate source. The contents of liver and hepatoma lipids ( $\mathrm{TCh}$ and $\mathrm{TG}$ ) were unaffected by Met addition with any carbohydrate source, except for hepatoma TG of the sucrose-starch diet-fed rats, which was slightly but significantly decreased by Met addition.
In experiment 3 , the Met action on serum lipids was examined in $\mathrm{Ch}$-loaded rats (Table IV). Methionine $(0.75 \%)$ exerted no significant influence on food intake, growth and relative liver weight, regardless of the dietary carbohydrate source. Unlike the normal and hepatoma-bearing states, Met modified the serum lipoprotein profiles when sucrose alone and starch alone were used as the dietary carbohydrates. Methionine notably reduced (VLDL + LDL)-Ch without affecting HDL$\mathrm{Ch}$, leading to significant decreases in TCh and AI, although AI of the sucrose-Met group had a decreasing tendency $(p<0.1)$ compared to the control group. The same trend was seen in the sucrose-starch-Met group, but none of the changes was statistically significant. The serum TG level was significantly reduced by Met addition with all dietary carbohydrate sources. The liver TCh content was unaffected by Met addition, while Met significantly elevated the liver TG content with all carbohydrate sources. 
Table 1II. Effect of Methionine (1.2\%) Addition to the 20\% Casein Diets Containing Sucrose, Starch or a Sucrose-Starch Mixture on the Food Intake, Body Weight Gain, Liver and Hepatoma Weights, Lipid Levels of Serum, Liver and Hepatoma in HEPATOMA-BEARING DONRYU RATS (EXPERIMENT 2)

\begin{tabular}{|c|c|c|c|c|c|c|}
\hline \multirow{3}{*}{ Measurement } & \multicolumn{6}{|c|}{ Dietary carbohydrate source } \\
\hline & \multicolumn{2}{|c|}{ Sucrose } & \multicolumn{2}{|c|}{ Starch } & \multicolumn{2}{|c|}{ Sucrose-starch } \\
\hline & Control & + Met & Control & + Met & Control & + Met \\
\hline No. of rats & 5 & 6 & 6 & 6 & 6 & 6 \\
\hline Initial body weight $(\mathrm{g})$ & $123.9 \pm 5.8$ & $122.1 \pm 4.9$ & $121.8 \pm 4.5$ & $121.4 \pm 3.5$ & $121.6 \pm 5.0$ & $121.7 \pm 4.8$ \\
\hline Food intake (g/2 weeks) & $126.5 \pm 14.9$ & $129.3 \pm 11.4$ & $138.8+9.2$ & $142.6 \pm 9.4$ & $134.3 \pm 7.3$ & $120.4 \pm 10.6$ \\
\hline Body weight gain ( $g / 2$ weeks) & $21.2 \pm 12.3$ & $32.9 \pm 8.5$ & $29.4 \pm 7.0$ & $30.6 \pm 10.0$ & $31.9 \pm 5.1$ & $22.0 \pm 7.8$ \\
\hline $\begin{array}{l}\text { Liver weight } \\
\qquad(\mathrm{g} / 100 \mathrm{~g} \text { of body weight })\end{array}$ & $4.15 \pm 0.28$ & $3.97 \pm 0.17$ & $3.74 \pm 0.13$ & $4.05 \pm 0.14$ & $4.12 \pm 0.12$ & $3.67 \pm 0.22$ \\
\hline $\begin{array}{l}\text { Hepatoma weight } \\
(\mathrm{g} / 100 \mathrm{~g} \text { of body weight })\end{array}$ & $11.30 \pm 1.75$ & $11.25 \pm 1.46$ & $10.23 \pm 1.78$ & $7.94 \pm 1.23$ & $12.79 \pm 1.36$ & $10.47 \pm 1.31$ \\
\hline \multicolumn{7}{|c|}{ Serum lipid $(\mathrm{mg} / 100 \mathrm{ml}$ of serum) } \\
\hline Triglyceride & $81.1 \pm 13.1$ & $84.7 \pm 9.4$ & $64.8 \pm 7.8$ & $65.5 \pm 6.4$ & $89.5 \pm 11.9$ & $54.2 \pm 7.9^{*}$ \\
\hline Total cholesterol & $117.5 \pm 5.4$ & $112.9 \pm 4.3$ & $111.3 \pm 8.7$ & $111.5 \pm 6.1$ & $121.6 \pm 2.7$ & $115.0 \pm 2.9$ \\
\hline $\mathrm{HDL}-\mathrm{Ch}(\mathrm{A})$ & $42.6 \pm 2.7$ & $46.8 \pm 4.3$ & $42.0 \pm 2.0$ & $42.5 \pm 1.9$ & $39.5 \pm 2.1$ & $47.2 \pm 2.2^{*}$ \\
\hline$(\mathrm{VLDL}+\mathrm{LDL})-\mathrm{Ch}(\mathrm{B})$ & $74.8 \pm 3.4$ & $66.1 \pm 3.4$ & $69.4 \pm 7.6$ & $69.0 \pm 4.6$ & $81.6 \pm 4.5$ & $67.8 \pm 2.8^{*}$ \\
\hline Atherogenic index $(B / A)$ & $1.77 \pm 0.08$ & $1.47 \pm 0.16$ & $1.65 \pm 0.15$ & $1.62 \pm 0.06$ & $2.12 \pm 0.23$ & $1.46 \pm 0.11^{*}$ \\
\hline \multicolumn{7}{|l|}{ Liver lipid ( $\mathrm{mg} / \mathrm{g}$ of liver) } \\
\hline Total cholesterol & $2.67 \pm 0.20$ & $2.84 \pm 0.45$ & $3.03 \pm 0.12$ & $3.10 \pm 0.18$ & $2.41 \pm 0.28$ & $2.77 \pm 0.31$ \\
\hline Triglyceride & $7.10 \pm 1.83$ & $13.18 \pm 2.67$ & $12.43 \pm 1.87$ & $15.87 \pm 5.37$ & $8.93 \pm 1.81$ & $6.50 \pm 1.56$ \\
\hline \multicolumn{7}{|c|}{ Hepatoma lipid (mg/g of hepatoma) } \\
\hline Total cholesterol & $1.90 \pm 0.15$ & $1.80 \pm 0.06$ & $1.81 \pm 0.08$ & $1.81 \pm 0.08$ & $1.97 \pm 0.07$ & $1.77 \pm 0.09$ \\
\hline Triglyceride & $3.78 \pm 0.31$ & $4.74 \pm 1.07$ & $4.31 \pm 1.06$ & $3.74 \pm 0.28$ & $4.45 \pm 0.24$ & $3.46 \pm 0.12 * *$ \\
\hline
\end{tabular}

Each value represents the mean $\pm \mathrm{SEM}$.

$* p<0.05$ and ${ }^{* *} p<0.005$ to the corresponding control groups.

\section{Discussion}

In the present study, the effect of a Met supplement to $20 \%$ casein diets on the serum Ch concentration and lipoprotein profiles was examined by varying the dietary carbohydrate source and hypercholesterolemic models. As animals for an endogenous hypercholesterolemic model, hepatoma-bearing rats ${ }^{10,11)}$ were employed. Since the ascites hepatoma line of AH109A used here originated from a rat of the Donryu strain, ${ }^{16)}$ rats of this strain were also employed in the other two experiments to strictly compare the effect of dietary carbohydrate on the Met action among three different metabolic states. To avoid possible changes in the genetic and biological characteristics of the rats due to farm differences, which have been indicated even in a single strain, ${ }^{16)}$ the rats were always obtained from the same farm of one supplier.

A suppression of food intake and growth by the Met supplement was observed only in normal rats (Table II). This may have been due to a larger amount $(1.8 \%)$ of added Met than those of the hepatoma-bearing $(1.2 \%)$ and Ch-loaded $(0.75 \%)$ rats (Tables III and IV).

From the present results, dietary carbohydrates are suggested to modify the Met action on serum $\mathrm{Ch}$ and lipoprotein profiles depending on the absence or presence of dietary $\mathrm{Ch}$. When $\mathrm{Ch}$-free, $20 \%$ casein diets were fed to both the normal and hepatoma-bearing rats, the Met action on the serum $\mathrm{Ch}$ and lipoprotein profiles was significant in the case of the sucrose-starch mixture. Under the dietary condition of Ch-enrichment, the Met action appeared definitely when sucrose alone or starch 
Table IV. Effect of Methionine $(0.75 \%$ ) Addition to the 20\% Casein Diets Containing Sucrose, Starch or a Sucrose-Starch Mixture on the Food Intake, Body Weight Gain, Liver Wejght, and Serum and Liver Lipid Levels in Cholesterol-loaded Donryu Rats (Experiment 3)

\begin{tabular}{|c|c|c|c|c|c|c|}
\hline \multirow{3}{*}{ Measurement } & \multicolumn{6}{|c|}{ Dietary carbohydrate source } \\
\hline & \multicolumn{2}{|c|}{ Sucrose } & \multicolumn{2}{|c|}{ Starch } & \multicolumn{2}{|c|}{ Sucrose-starch } \\
\hline & Control & + Met & Control & + Met & Control & + Met \\
\hline No. of rats & 5 & 5 & 5 & 5 & 5 & 5 \\
\hline Initial body weight $(\mathrm{g})$ & $119.8 \pm 2.5$ & $120.0 \pm 2.6$ & $119.8 \pm 3.3$ & $121.4 \pm 4.7$ & $119.8 \pm 4.0$ & $119.8 \pm 3.6$ \\
\hline Food intake ( $\mathrm{g} / 2$ weeks $)$ & $150.8 \pm 5.8$ & $144.5 \pm 8.7$ & $160.8 \pm 9.1$ & $153.3 \pm 5.1$ & $168.8 \pm 12.6$ & $144.0 \pm 11.9$ \\
\hline Body weight gain ( $\mathrm{g} / 2$ weeks) & $47.6 \pm 7.4$ & $54.5 \pm 9.4$ & $44.1 \pm 8.3$ & $62.9 \pm 6.1$ & $60.2 \pm 8.1$ & $53.3 \pm 8.8$ \\
\hline $\begin{array}{l}\text { Liver weight } \\
\qquad(\mathrm{g} / 100 \mathrm{~g} \text { of body weight })\end{array}$ & $5.35 \pm 0.13$ & $5.47 \pm 0.15$ & $4.90 \pm 0.15$ & $4.74 \pm 0.16$ & $4.77 \pm 0.11$ & $5.09 \pm 0.15$ \\
\hline \multicolumn{7}{|c|}{ Serum lipid $(\mathrm{mg} / 100 \mathrm{ml}$ of serum $)$} \\
\hline Triglyceride & $49.6 \pm 2.6$ & $32.4 \pm 1.8^{* *}$ & $51.0 \pm 4.1$ & $30.0 \pm 1.8^{* *}$ & $50.8 \pm 5.1$ & $36.7 \pm 3.3^{*}$ \\
\hline Total cholesterol & $227.8 \pm 39.0$ & $102.9 \pm 17.0^{*}$ & $143.8 \pm 21.4$ & $83.2 \pm 7.3^{*}$ & $115.8 \pm 7.4$ & $88.9 \pm 12.5$ \\
\hline $\mathrm{HDL}-\mathrm{Ch}(\mathrm{A})$ & $45.7 \pm 7.2$ & $57.9 \pm 5.6$ & $48.1 \pm 4.1$ & $56.9 \pm 3.7$ & $52.7 \pm 3.5$ & $49.7 \pm 5.0$ \\
\hline$(\mathrm{VLDL}+\mathrm{LDL})-\mathrm{Ch}(\mathrm{B})$ & $182.1 \pm 45.1$ & $45.0 \pm 20.2^{*}$ & $95.8 \pm 23.6$ & $26.3 \pm 7.8^{*}$ & $63.1 \pm 10.5$ & $39.3 \pm 13.2$ \\
\hline Atherogenic index $(B / A)$ & $5.50 \pm 2.17$ & $0.96 \pm 0.54$ & $2.21 \pm 0.65$ & $0.49 \pm 0.18^{*}$ & $1.27 \pm 0.25$ & $0.91 \pm 0.41$ \\
\hline \multicolumn{7}{|l|}{ Liver lipid ( $\mathrm{mg} / \mathrm{g}$ of liver) } \\
\hline Total cholesterol & $64.2 \pm 3.0$ & $62.1 \pm 2.1$ & $64.1 \pm 4.3$ & $61.5 \pm 4.6$ & $63.8 \pm 3.9$ & $58.7 \pm 4.9$ \\
\hline Triglyceride & $65.5 \pm 7.3$ & $112.8 \pm 13.4^{*}$ & $58.7 \pm 2.7$ & $83.9 \pm 10.6^{*}$ & $67.9 \pm 5.0$ & $97.4 \pm 11.0^{*}$ \\
\hline
\end{tabular}

Each value represents the mean \pm SEM.

${ }^{*} p<0.05$ and $* * p<0.005$ to the corresponding control groups.

alone was used as the dietary carbohydrate source. There is, at present, no pertinent explanation for these effects of sucrose, starch and their mixture on the Met action. One possible reason is that the response of intestinal flora to dietary carbohydrates and/or the digestion and hence absorption of carbohydrates might be different between the absence and presence of $\mathrm{Ch}$, cholate and high fat in the diets, such a difference in the intestine possibly modifying the Met action through an unknown mechanism.

In the normal and Ch-loaded states, there is no essential abnormality in lipid metabolism, and the regulatory function of $\mathrm{Ch}$ metabolism is thought to be fully operating in the Chloaded state. ${ }^{17)}$ By contrast, the regulatory function is fundamentally destroyed in such secondary hypercholesterolemic states ${ }^{17)}$ as the hypothyroid ${ }^{91}$ and hepatoma-bearing ${ }^{10)}$ ones. In the present study, the two contrasting states of Ch-loaded and hepatoma-bearing were employed together with the normal state to estimate the Met action on the serum $\mathrm{Ch}$ and lipoprotein profiles by varying the carbohydrate source, using a single strain (Donryu) of rats obtained from a single farm. Whenever a significant action of Met was observed on the serum $\mathrm{Ch}$ and lipoprotein profiles, this amino acid reduced $\mathrm{AI}$ by increasing $\mathrm{HDL}-\mathrm{Ch}$ (sucrose-starch mixture in Table II), by decreasing (VLDL $+\mathrm{LDL}$ )-Ch (sucrose alone or starch alone in Table IV) or by both actions (sucrose-starch mixture in Table III). These results indicate that Met essentially acts as an antiatherogenic amino acid in respect of $\mathrm{AI}$ in rats of the Donryu strain under all three metabolic conditions examined here. It has been pointed out that the response of serum Ch and lipoproteins to dietary-added Met was different among strains of rats in the normal and $\mathrm{Ch}$-loaded states. ${ }^{2)}$ Methionine has been reported to increase the HDL-Ch level in normocholesterolemic Wistar rats, as was seen in the normocholesterolemic Donryu rats (sucrose-starch mixture in Table II), by adding $0.75 \%$ and $1.5 \%$ of this amino acid to a $20 \%$ casein $\operatorname{diet}^{6)}$ or $1.0 \%$ to a $25 \%$ casein diet, ${ }^{8}$ 
both of which contained a sucrose-starch $(1: 2)$ mixture as the carbohydrate source. In a separate experiment, Met $(1.5 \%)$ exerted no influence on the serum $\mathrm{Ch}$ and HDL-Ch levels in normocholesterolemic Wistar rats fed on a $20 \%$ casein diet containing a sucrose-starch (1:3) mixture (data not shown). From these results with Wistar rats and the indication of variation in the genetic and biological characteristics even in a single strain, ${ }^{16)}$ differences in the farm source even within the same strain might be also considered as another possible reason for the varying effect of Met on serum $\mathrm{Ch}$ and lipoproteins in the normocholesterolemic and probably Ch-loaded states, in addition to the effect from strain differences. Thus, it seems meaningful to artificially modify a strong $\mathrm{Ch}$ homeostasis in rats ${ }^{17 \text { ) }}$ like hepatoma transplantation. This would remove the varying response of serum $\mathrm{Ch}$ and lipoproteins to dietary-supplemented Met, which might be due to differences in the genetic background.

Lipoprotein lipase (LPL) ${ }^{18)}$ and hepatic TG lipase (HTGL) ${ }^{19,20)}$ have been well documented to affect the catabolism of TG-rich lipoproteins. Furthermore, Nikkilä et al. ${ }^{21)}$ have reported the presence of a positive correlation between adipose tissue LPL activity and HDL-Ch concentration. Patsch et al. ${ }^{22}$ have demonstrated the formation of an $\mathrm{HDL}_{2}$-like lipoprotein by incubating in vitro VLDL and $\mathrm{HDL}_{3}$ with purified LPL. These findings indicate that LPL affected the quantity and quality of HDL, as well as the catabolism of TG-rich lipoproteins. In hepatoma-bearing Donryu rats, the serum TG and (VLDL+ LDL)-Ch levels have been shown to be higher and the HDL-Ch level to be lower than in normal rats. ${ }^{10)}$ Methionine reduced the hepatoma-induced elevation of serum TG and $(\mathrm{VLDL}+\mathrm{LDL})-\mathrm{Ch}$, and prevented the hepatoma-induced decrease of $\mathrm{HDL}-\mathrm{Ch}$ (sucrosestarch mixture in Table III). In mice bearing a GRSL ascites tumor (a murine leukemia model), the LPL activity has been reported to have decreased. ${ }^{23)}$ Thus, LPL activity is presumably less in the hepatoma-bearing state. Measurements of the activity of LPL together with HTGL will clarify this aspect and also inform us whether or not Met is concerned with these TG lipase activities.

In Ch-loaded Donryu rats, the serum TG level was decreased and the liver TG content was inversely increased by Met with all the dietary carbohydrate sources used (Table IV). A suppression of VLDL formation and/or its secretion in the liver may be one possible cause of the hypolipidemic and hepatic TGaccumulative action of Met in Ch-loaded Donryu rats, since a reduction of VLDL formation and/or secretion sometimes accompanies a TG accumulation in the liver. ${ }^{24)}$

From the results obtained here, the Met action on the serum $\mathrm{Ch}$ and lipoprotein profiles is suggested to be modified by dietary carbohydrates. The supplemented levels of Met, however, were not identical among the three experiments. Therefore, further studies by changing the supplemental Met level are needed to obtain more information for comparing exactly the Met action at the same level of this amino acid among the three different cholesterolemic and dietary carbohydrate conditions. Studies concerning the mechanism by which Met exerts lipid metabolism are also needed to learn if the mode of action of Met is common or not among the three different states employed here.

\section{References}

1) E. G. Hill, J. Nutr., 89, 143 (1966).

2) K. Tsuji, T. Ichikawa, M. Kawamura, Y. Nakagawa and M. Inomata, Sulfur Amino Acids, 8, 489 (1985).

3) K. Sugiyama, Y. Kushima and K. Muramatsu, Agric. Biol. Chem., 49, 3455 (1985).

4) K. Yagasaki, T. Aoki and R. Funabiki, Nutr. Rep. Int, 34, 59 (1986).

5) A. H. M. Terpstra, R. J. J. Hermus and C. E. West, in "Animal and Vegetable Proteins in Lipid Metabolism and Atherosclerosis," ed. by M. J. Gibney and D. Kritchevsky, Alan R. Liss Inc., New York, 1983, pp. 19 49.

6) S. Nagaoka, Y. Aoyama and A. Yoshida, Nutr. Rep. Int., 31, 1137 (1985).

7) C. Sérougne and A. Rukaj, Ann. Nutr. Metab., 27, 386 (1983).

8) E. Ohmura, T. Ishikawa, M. Takagi, Y. Aoyama and 
A. Yoshida, Agric. Biol. Chem., 52, 1027 (1988).

9) K. Yagasaki, T. Aoki, M. Machida and R. Funabiki, Agric. Biol. Chem., 50, 2785 (1986).

10) K. Yagasaki, M. Machida and R. Funabiki, J. Nutr. Sci. Vitaminol., 32, 643 (1986).

11) T. Irikura, K. Takagi, K. Okada and K. Yagasaki, Lipids, 20, 420 (1985).

12) J. Folch, M. Lees and G. H. Sloane-Stanley, J. Biol. Chem., 226, 497 (1957).

13) B. Zak, Am. J. Clin. Path., 27, 583 (1957).

14) E. Van Handel, Clin. Chem., 7, 249 (1961).

15) K. Yagasaki, K. Okada, K. Takagi and T. Irikura, Agric. Biol. Chem., 48, 1417 (1984).

16) H. Sato, Igaku to Yakugaku, 4, 587 (1980).

17) N. Takeuchi, Nippon Rinshō, 30, 347 (1972).

18) A. Cryer, S. E. Riley, E. R. Williams and D. S.
Robinson, Biochem. J., 140, 561 (1974).

19) J. Grosser, O. Schrecker and H. Greten, J. Lipid Res., 22, 437 (1981).

20) T. Murase and H. Itakura, Atherosclerosis, 39, 293 (1981).

21) E. A. Nikkilä, M.-R. Taskinen and M. Kekki, Atherosclerosis, 29, 497 (1978).

22) J. R. Patsch, A. M. Gotto, Jr., T. Olivecrona and S. Eisenberg, Proc. Natl. Acad. Sci. U.S.A., 75, 4519 (1978).

23) J. Damen, J. Van Ramshorst, R. P. Van Hoeven and W. J. Van Blitterswijk, Biochim. Biophys. Acta, 793, 287 (1984).

24) K. Yagasaki and M. Kametaka, Nutr. Rep. Int., 29, 533 (1984). 